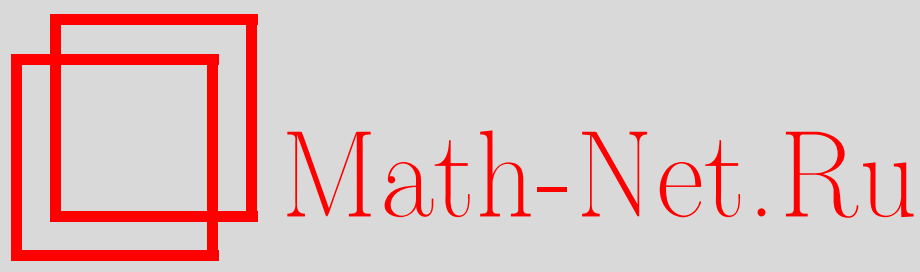

А. А. Туганбаев, Модули над наследственными кольцами, Матем. сб., 1998, том 189, номер 4, 145-160

DOI: https://doi.org/10.4213/sm316

Использование Общероссийского математического портала Math-Net.Ru подразумевает, что вы прочитали и согласны с пользовательским соглашением

http: //www. mathnet.ru/rus/agreement

Параметры загрузки:

IP : 54.198 .187 .58

26 апреля 2023 г., 12:36:41 
УДК 512.55

\author{
А.А. Туганбаев
}

\title{
Модули над наследственными кольцами
}

Пусть $A$ - наследственное нётерово первичное кольцо, не являющееся примитивным справа. Получено полное описание $\pi$-инъективных $A$-модулей. Выяснено, когда классическое кольцо частных колца $A$ является $\pi$-проективньм $A$-модулем.

Получен критерий того, что наследственное справа нётерово справа первичное колцц является полуцепньм кольцом.

Библиографиял: 8 названий.

Все кольца, встречающиеся в работе, предполагаются ассоциативными и с ненулевой единицей. Слова типа "нётерово кольцо" означают, что соответствующие условия выполнены справа и слева. Через $J(M)$ обозначается радикал Джекобсона модуля $M$. Модуль называется наследственным, если все его подмодули проективны. Модуль назьвается цепным, если любые его два подмодуля сравнимы по включению. Прямая сумма цепных модулей называется полуцепным модулем. Цепная нётерова область $A$ назьвается полной, если кольцо $A$ является полным относительно $J(A)$-адической топологии.

Модуль $M$ назьвается проективным относительно модуля $N$ (или $N$-nроективным), если для любого эпиморфизма $h: N \rightarrow \bar{N}$ и каждого гомоморфизма $\bar{f}: M \rightarrow \bar{N}$ существует такой гомоморфизм $f: M \rightarrow N$, что $h f=\bar{f}$. Модуль, проективный относительно себя, называется квазипроективным (или самопроективнылм) модулем. Модуль $M$ называется $\pi$-проективным (см. [1, с. 359]), если для любых его подмодулей $U$ и $V$ таких, что $U+V=M$, сушествует такой эндоморфизм $f$ модуля $M$, что $f(M) \subseteq U,(1-f)(M) \subseteq V$. Если все фактормодули модуля $M$ являются неразложимьми, то $M$ является, очевидно, $\pi$-проективньм модулем. Поэтому все цепные модули являются $\pi$-проективньми. Каждый квазипроективный модуль является $\pi$-проективным [1, с. 359]. Любая квазициклическая абелева группа $C\left(p^{\infty}\right)$ является $\pi$-проективным, но не квазипроективным модулем над кольцом целых чисел.

Напомним, что каждое нётерово первичное кольцо $A$ обладает простым артиновьм классическим кольцом частных $Q$, причем $Q_{A}$ является инъективным несингулярным правым (левым) $A$-модулем. В [2, теорема 8] выяснено, когда классическое кольцо частных наследственного нётерова первичного кольца $A$, не являющегося примитивным справа, является квазипроективным правьм $A$-модулем. В связи этим отметим первые два основных результата данной работы - теоремы 1 и 2 .

ТеОРема 1. Пусть $A$ - наследственное справа нётерово справа первичное кольцо. Тогда равносильны следующие условия:

(1) существует ненулевой инбективный $\pi$-проективный правый $A$-модуль $M$, не являющийся сингулярным;

(2) $A$ - полуцепное кольцо.

Работа вьполнена при поддержке Российского фонда фундаменталњных исследований (грант № 96-01-00627). 
ТЕОРЕма 2. Пусть $Q$ - классическое кольцо частных наследственного нётерова первичного кольиа А. Тогда равносильны следующие условия:

(1) $Q-\pi$-проективный правъьй $A$-модуль;

(2) либо $A$ - цепная нётерова область, либо существует такое натуральное число $n$, что кольцо $A$ изоморфно кольиу всех $(n \times n)$-матрии, над некоторой полной цепной нётеровой областью $D$.

Сушествуют цепные нётеровы области $A$, не являющиеся ни примитивными справа, ни полньми (например, в качестве $A$ можно взять кольцо всех рациональных чисел с нечетными знаменателями). Поэтому из теоремы 2 следует, что существуют наследственные нётеровы первичные кольца $A$, не являющиеся примитивными справа, у которых классические кольца частных являются $\pi$-проективньми, но не квазипроективными правыми $A$-модулями. Следовательно, даже в непримитивном случае, существуют кольца, подпадающие под теорему 2 и не подпадающие под вьшшеупомянутую теорему 8 из [2].

Модуль $M$ назьвается инвективным относительно модуля $N$ (или $N$-инбективным), если для любого подмодуля $\bar{N}$ модуля $N$ каждый гомоморфизм $\bar{f}: \bar{N} \rightarrow M$ может быть продолжен до гомоморфизма $f: N \rightarrow M$. Модуль, инъективный относительно себя, называется квазиинғективным (или самоинғективным) модулем. Модуль $M$ называется $\pi$-инғективным (см. [1, с. 367]), если для любых его подмодулей $U$ и $V$ таких, что $U \cap V=0$, существует такой эндоморфизм $f$ модуля $M$, что $f(U)=0,(1-f)(V)=0$. Модуль называется равномерным, если все его подмодули являются неразложимыми. В частности, все цепные модули являются $\pi$-инъективными. Каждый квазиинъективньй модуль является $\pi$-инъективньм. Бесконечная циклическая группа является $\pi$-инъективным, но не квазиинъективным, равномерным модулем над кольцом целых чисел.

В [2, теорема 7] получено полное описание квазиинъективных правых модулей над ограниченньми наследственными нётеровыми первичньпи кольцами. В связи с этим мы докажем третий основной результат работы - теорему 3.

ТЕОРема 3. Пусть $M_{A}-$ модуль над наследственным нётеровым первичным кольцом $A$, причем $А$ не является примитивным справа. Тогда равносильны следующие условия:

(1) $M-\pi$-инвективный модуль;

(2) либо $M$ - инбективный модуль, либо $M$ - квазиинбективный сингулярный модуль, либо $M=T \oplus U$, где $T$ - инвективный сингулярный модуль, $U-$ равномерный несингулярный модуль.

Модуль, не имеющий ненулевых инъективных подмодулей, называется редуцированным. Модуль, не содержащий бесконечных прямых сумм ненулевых подмодулей, называется конечномерным (в смысле Голди). Четвертым основным результатом работы является полученный в теореме 4 критерий проективности.

ТЕОРема 4. Пусть $M_{A}-$ модуль над наследственным нётеровым первичным кольиом $A$, причем $А$ не является примитивным справа кольцом. Тогда равносильны следующие условия:

(1) $M_{A}$ - конечнопорожденный проективный модуль;

(2) $M-\pi$-проективный редуцированный конечномерный несингулярный модуль. 
Заметим, что в теореме 4 нельзя опустить условие редуцированности. Действительно, пусть $A$ - кольцо всех рациональных чисел с нечетными знаменателями, $Q-$ поле частных коммутативной цепной нётеровой наследственной области $A$. Тогда $Q_{A}-\pi$-проективный конечномерный несингулярный модуль, не являющийся проективным.

Доказательство теорем разбито на ряд лемм, сгруппированных в два параграфа. Приведем ряд необходимых определений и обозначений. Модуль называется вполне индективныц, если все его фактормодули инъективны. Подмодуль фактормодуля модуля $M$ называется подфактором модуля $M$. Через $\operatorname{End}(M)$ и $\operatorname{Lat}(M)$ обозначаются, соответственно, кольцо эндоморфизмов и решетка подмодулей модуля $M$. Через $r_{A}(N)$ обозначается аннулятор в кольце $A$ подмножества $N$ правого модуля $M_{A}$, причем индекс может опускаться, когда ясно, какое кольцо имеется в виду. Через $\operatorname{Sing}(M)$ обозначается сингулярный подмодуль модуля $M_{A}$ над кольцом $A$ (т.е. множество всех таких элементов $m \in M$, что $r(m)-$ сушественный правый идеал кольца $A$ ). Модуль $M_{A}$ называется несингулярным (сингулярным $)$, если $\operatorname{Sing}(M)=0(\operatorname{Sing}(M)=M)$. Элемент $а$ кольца $A$ называется регулярным, если $r(a)=\ell(a)=0$, где $\ell(a)$ - левый аннулятор элемента $a \in A$. Модуль $M_{A}$ называется делимым, если $M a=M$ для любого регулярного элемента $a \in A$. Через $\mathrm{T}(M)$ обозначается периодическая часть модуля $M_{A}$ (т.е. множество всех таких элементов $m \in M$, что аннулятор $r(m)$ содержит регулярный элемент кольца $A$ ). Модуль $M_{A}$ назьвается периодическим (модулем без кручения), если $\mathrm{T}(M)=M(\mathrm{~T}(M)=0)$. Прямая сумма равномерных модулей называется полуравномерным модулем. Конечномерное справа кольцо с условием максимальности для правых аннуляторов называется правым кольцом Голди. Модуль, у которого все циклические подмодули проективны, называется риккартовым модулем. Модуль $M$ называется конечно точным, если существует такое натуральное число $n$, что прямая сумма $M^{n}$ изоморфных копий модуля $M$ содержит свободный циклический подмодуль. Модуль называется полуартиновыц, если каждый его ненулевой фактормодуль является существенным расширением полупростого модуля. Модуль $M$ называется дистрибутивныцм, если $\operatorname{Lat}(M)$ - дистрибутивная решетка (т.е. $X \cap(Y+Z)=X \cap Y+X \cap Z$ для любых подмодулей $X, Y, Z$ модуля $M)$. Кольцо $A$ называется ограниченным справа, если каждый его сушественный правый идеал содержит ненулевой идеал кольца $A$. Кольцо $A$ называется совершенным справа, если $A / J(A)$ - артиново кольцо и для любой последовательности $\left\{a_{i}\right\}_{i=1}^{\infty}$ элементов из $J(A)$ существует такой номер $n$, что $a_{n} \cdot \ldots \cdot a_{1}=0$.

\section{§1. Несингулярные инъективные модули и полуцепные кольца}

ЛЕмма 1.1. Пусть $A$ - кольцо, $Q$ - индективная оболочка модуля $A_{A}, E_{A}$ - модуль, не являющийся сингуляныц. Тогда справедливо следующее:

(1) существует подмодуль $N$ модуля $E$, изоморфный ненулевому правому идеалу кольца $A$;

(2) если E - неразложимый инвективный модуль, то $E$ изоморфен некоторому неразложимому прямому слагаемому $S$ модуля $Q$.

ДокАЗАТЕЛЬСТво. По условию существует такой ненулевой элемент $m \in E$, что правый идеал $r(m)$ кольца $A$ не является существенным. Пусть $h: A_{A} \rightarrow m A$ - естественный эпиморфизм, $B$ - такой ненулевой правый идеал кольца $A$, что $B \cap r(m)=0$. Так как $B \cap \operatorname{Ker}(h)=0$, то модуль $B_{A}$ изоморфен ненулевому 
подмодулю $h(B)$ модуля $E$.

Допустим, что $E$ - неразложимый инъективный модуль. Тогда $E$ - инъективная оболочка модуля $h(B)$. Модуль $Q$ имеет прямое слагаемое $S$, являющееся инъективной оболочкой модуля $B$. Так как $h(B) \cong B_{A}$, то $S \cong E$. Поэтому $S$ - неразложимый модуль.

Лемма 1.2. (1) $U$ - несингулярный модуль $\Longleftrightarrow \operatorname{Hom}(T, U)=0$ для любого сингулярного модуля $T \Longleftrightarrow$ любое существенное расширение модуля $U$ является несингулярным модулем;

(2) если $N$ - существенный подмодуль модуля $Q$, то $Q / N-$ сингулярный модуль;

(3) если $N$ - ненулевой подмодуль равномерного модуля $Q$, то $Q / N$ сингулярный модуль;

(4) если $f: Q \rightarrow E-$ гомоморфизм модулей и $\operatorname{Ker}(f)$ - существенный подмодуль модуля $Q$, то $f(Q)$ - сингулярный модуль;

(5) если $E$ - несингулярный модуль и $f: Q \rightarrow E$ - ненулевой модульныи гомоморфизм, то $Q$ не является существенным расширением модуля $\operatorname{Ker}(f)$;

(6) если $E_{1}, E_{2}$ - несингулярные равномерные модули, то любой ненулевой гомоморфизм $E_{1} \rightarrow E_{2}$ является мономорфизмом;

(7) если $E_{1}, E_{2}$ - несингулярные неразложсмие инбективные модули, то любой ненулевой гомоморфизм $E_{1} \rightarrow E_{2}$ является изоморфизмом.

ДокАЗАТЕЛьство. Пункты (1) и (2) доказаны в [3, 1.20, 1.22]; (3) следует из (2) и того, что равномерный модуль является сушественным расширением любого своего ненулевого подмодуля; (4) следует из (2) и того, что $f(Q) \cong Q / \operatorname{Ker}(f)$; (5) следует из (4); (6) следует из (5); (7) следует из (6).

Лемма 1.3. Пусть $A$ - первичное кольцо, $Q$ - инвективная оболочка модуля $A_{A}, \mathscr{E}$ - множсество всех ненулевых несингулярных неразложсимых инбективных правых А-модулей. Тогда справедливо следующее:

(1) $\operatorname{Hom}\left(B_{A}, C_{A}\right) \neq 0$ для любых ненулевых правых идеалов $B, C$ кольца $A$;

(2) если $B_{1}$ и $B_{2}$ - ненулевые правые идеаль кольца $A, S_{1}$ и $S_{2}$ - инвективные оболочки правых $A$-модулей $B_{1}$ и $B_{2}$, лежсащие в модуле $Q$, mo $\operatorname{Hom}\left(S_{1}, S_{2}\right) \neq 0$;

(3) либо множество $\mathscr{E}$ является пустым, либо существует такое ненулевое несингуярное неразлохимое инғективное прямое слагаемое $S$ модуля $Q$, что каждый модуль $E$ из $\mathscr{E}$ изоморфен модулю $S$;

(4) если $B, C$ - ненулевые несингулярные равномерные правые идеаль кольиа $A$, то существуют мономорфизмь $B \rightarrow C u C \rightarrow B$.

ДоказАтельство. (1) Так как $A$ - первичное кольцо, то $C B \neq 0$. Поэтому $c B \neq 0$ для некоторого элемента $c \in C$. Тогда правило $f(b)=c b$ задает ненулевой гомоморфизм $f: B \rightarrow C$.

(2) Следует из (1) и того, что любой гомоморфизм $B_{1} \rightarrow B_{2}$ может быть продолжен до гомоморфизма $S_{1} \rightarrow S_{2}$.

(3) Пусть $E_{1}, E_{2} \in \mathscr{E}$. По лемме $1.1(2) E_{i} \cong S_{i}$, где $S_{i}$ - ненулевое несингулярное неразложимое инъективное прямое слагаемое модуля $Q, i=1,2$. По (2) $\operatorname{Hom}\left(S_{1}, S_{2}\right) \neq 0$. По лемме $1.2(7) S_{1} \cong S_{2}$.

(4) Следует из (1) и из леммы $1.2(6)$. 
Лемма 1.4. Пусть Е-существенное расширение модуля $M$. Тогда справедливо следующее:

(1) если $N-$ модуль, $f \in \operatorname{Hom}(N, E)$ и существует такой гомоморфизм $g: N \rightarrow M$, ито $f$ совпадает с $g$ на $f^{-1}(M)$, то $f(N) \subseteq M$;

(2) если модуль $M$ ингективен относительно модуля $N$, то $f(N) \subseteq M$ для любого гомоморфизма $f \in \operatorname{Hom}(N, E)$;

(3) если $f$ - эндоморфизм модуля $E$ и существует такой эндоморфизм $g$ модуля $M$, что $f$ совпадает с $g$ на $M \cap f^{-1}(M)$, mo $f(M) \subseteq M$.

Доказательство. (1) Пусть $y=(f-g)(x) \in M \cap(f-g)(N), x \in N$. Тогда $f(x)=(f-g)(x)+g(x)=y+g(x) \in M$. Поэтому $x \in f^{-1}(M)$. Но тогда $y \in(f-g)\left(f^{-1}(M)\right)=0$. Поэтому $M \cap(f-g)(N)=0$. Так как $E-$ существенное расширение модуля $M$, то $(f-g)(N)=0$. Тогда $f(N)=g(N) \subseteq M$.

(2) Так как $f\left(f^{-1}(M)\right) \subseteq M$ и $M-N$-инъективный модуль, то существует такой гомоморфизм $g: N \rightarrow M$, что $g$ совпадает с $f$ на $f^{-1}(M)$. Из (1) следует вложение $f(N) \subseteq M$.

(3) Следует из (1).

Лемма 1.5. Пусть Е- инбективная оболочка модуля М. Равносильньц следующие условия:

(1) $M-\pi$-инбективный модуль;

(2) каждый идемпотентный әндоморфизм любого подмодуля модуля $M$ может бъть продолжен до әндоморфизма модуля $M$;

(3) каждый идемпотентный әндоморфизм любого подмодуля модуля $M$ может быть продолжен до идемпотентного әндоморфизма модуля $M$

(4) для любъх подмодулей $N_{1}, N_{2}$ модуля $M$ mаких, что $N_{1} \cap N_{2}=0$, существует такое прямое разлохсение $M=M_{1} \oplus M_{2}$, что $M_{1} \supseteq N_{1}$, $M_{2} \supseteq N_{2}$

(5) $f(M) \subseteq M$ для любого идемпотентного эндоморфизма $f$ модуля $E$;

(6) для любого прямого разложсения $E=\bigoplus_{i \in I} E_{i}$ модуля $E$ верно равенство $M=\bigoplus_{i \in I}\left(M \cap E_{i}\right)$.

ДокАЗАТЕльСтво. Эквивалентность $(1) \Longleftrightarrow(2)$ и импликации $(4) \Longrightarrow(3)$, $(3) \Longrightarrow(2),(5) \Longrightarrow(6)$ проверяются непосредственно.

$(2) \Longrightarrow(5)$. Пусть $N \equiv M \cap f^{-1}(M)$. Так как $f^{2}=f$, то $f(N) \subseteq N$. Поэтому ограничение эндоморфизма $f$ на модуль $N$ является идемпотентным эндоморфизмом модуля $N$. По условию существует такой эндоморфизм $g$ модуля $M$, что $f$ совпадает с $g$ на $N$. Из леммы 1.4 (3) следует вложение $f(M) \subseteq M$.

$(6) \Longrightarrow(4)$. Пусть $N_{1} \oplus N_{2} \in \operatorname{Lat}(M)$. Так как $E$ - инъективный модуль, то существует такоепрямое разложение $E=E_{1} \oplus E_{2}$, что $E_{i}$ содержит $N_{i}$ при $i=1,2$. По условию $M=M_{1} \oplus M_{2}$, где $M_{i} \equiv E_{i} \cap M$ при $i=1,2$.

Лемма $1.6[1,17.11,19.9]$. Пусть $E$ - инбективная оболочка модуля $M$. Тогда справедливо следующее:

(1) $M-$ квазиинбективный модуль $\Longleftrightarrow M-$ вполне инвариантный подмодуль модуля $E$;

(2) если $M$ - квазиинбективный неразложимый модуль, то $M$ - равномерный модуль и $\operatorname{End}(M)$ - локальное кольцо. 


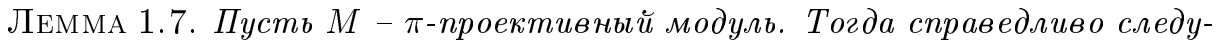
ющ,е :

(1) если $\operatorname{End}(M)$ - локальное кольцо, то все фактормодули модуля $M$ являются неразложимицми;

(2) если $\operatorname{End}(M)$ - локальное кольцо и все фактормодули модуля $M$ являются $\pi$-инбективными , то $M$ - иепной модуль;

(3) если $M$ - неразложимыи модуль, у которого все фактормодули квазиинбективнь, то $M-$ иепной модуль;

(4) если $M$ - неразложсимый вполне инбективный модуль, то $M-$ иепной модуль.

ДокаЗАТЕЛЬСТво. (1) Пусть $h: M \rightarrow \bar{M}$ - эпиморфизм, причем верно разложение $\bar{M}=h\left(M_{1}\right) \oplus h\left(M_{2}\right)$, где $M_{1}, M_{2} \in \operatorname{Lat}(M)$. Тогда $M=M_{1}+M_{2}$. Так как $M-\pi$-проективный модуль, то сушествует такой эндоморфизм $f \in \operatorname{End}(M)$, что $f(M) \subseteq M_{1},(1-f)(M) \subseteq M_{2}$. Так как $\operatorname{End}(M)$ - локальное кольцо, то либо $M_{1}=M$, либо $M_{2}=M$. Поэтому $\bar{M}$ - неразложимый модуль.

(2) Из леммы 1.5 следует, что каждый неразложимый $\pi$-инъективньй модуль является равномерным. Из (1) и из условия следует, что каждый фактормодуль модуля $M$ является равномерным. Поэтому $M$ - цепной модуль.

(3) Следует из (2) и леммы 1.6 (2).

(4) Следует из (3).

ЛЕмма 1.8. Пусть каждый неразложимый инвективный правый модуль над кольиом А является цепным. Тогда справедливо следующее:

(1) если $A$ - полущепное справа кольцо, то $A$ - полущепное кольцо;

(2) если $A$ - полуравномерное справа кольцо, то $A$ - полуцепное кольцо.

ДокАЗАТЕЛЬСтво. Пункт (1) доказан в [1, 55.11]; (2) следует из (1) и того, что инъективная оболочка любого равномерного модуля является равномерным модулем.

ЛЕмма 1.9. Пусть $E_{A}$ - ненулевой неразложсммый инвективный модуль над кольцом $A$, причем инвективная оболочка $Q_{A}$ модуля $A_{A}$ является вполне инвективным модулем. Тогда справедливо следующее:

(1) существует эпиморфизм $f: Q \rightarrow E u$, следовательно, $E$ - вполне инбективный модуль;

(2) если $Q=\bigoplus_{i \in I} Q_{i}$, то $E$ является гомоморфным образом хотя би одного из модулей $Q_{i}$;

(3) если $Q$ - прямая сумма неразложсимых $\pi$-проективных модулей, то кажсдый неразложимый инвективный правый $A$-модуль является иепным вполне инбективным модулем;

(4) если $Q$ - прямая сумма неразложимых $\pi$-проективных модулей и $A$ полуравномерное справа кольцо, то $A$ - полуцепное кольцо.

ДокаЗАтЕЛьство. (1) Пусть $\bar{E}$ - ненулевой циклический подмодуль модуля $E$. Сушествует ненулевой эпиморфизм $\bar{f}: A_{A} \rightarrow \bar{E} \subseteq E$. Так как $E$ - инъективный модуль, то $\bar{f}$ можно продолжить до ненулевого гомоморфизма $f: Q \rightarrow E$. Так как $Q$ - вполне инъективный модуль, то $f(Q)$ - ненулевой инъективный подмодуль неразложимого модуля $E$ и, следовательно, $f(Q)=E$.

(2) По (1) сушествует эпиморфизм $f: Q \rightarrow E$. Так как $E \neq 0$, то $f\left(Q_{i}\right) \neq 0$ для некоторого $i \in I$. По условию $f\left(Q_{i}\right)$ - инъективный модуль. Тогда $f\left(Q_{i}\right)-$ ненулевое прямое слагаемое неразложимого модуля $E$. Поэтому $E=f\left(Q_{i}\right)$. 
(3) Из лемм $1.7(4)$ и $1.9, Q$ - полуцепной модуль. Теперь утверждение следует из (2) и того, что все гомоморфные образы цепных модулей являются цепньми.

(4) Следует из (3) и леммы 1.8 (2).

Лемма 1.10 [4, с. 290]. Если кольцо А обладает самоинвективныцм справа классическим правым кольцом частных $Q$, то $Q_{A}$ - инбективная оболочка модуля $A_{A}$.

Лемма 1.11. Каждый риккартов модуль является несингулярным.

Лемма 1.11 проверяется непосредственно.

Лемма 1.12. Для кольиа А равносильны следующие условия:

(1) А обладает полупростымм артиновым классическим правым кольчом частных;

(2) A - полупервичное правое кольцо Голди;

(3) $A$ - несингулярное справа конечномерное справа полупервичное кольuо ;

(4) множество всех существенных правых идеалов кольца А совпадает с множсеством всех правых идеалов, содержсащих регулярные әлемен$m b l$

(5) А обладает полупростым артиновым классическим правым кольцом частных $Q$, причем $Q_{A}-$ индективная оболочка модуля $A_{A}$.

В лемме 1.12 эквивалентности $(1) \Longleftrightarrow(2) \Longleftrightarrow(3)$ доказаны в [5, следствие 9.13], эквивалентность $(2) \Longleftrightarrow(4)$ доказана в [4, теорема 2.2$]$, импликация $(1) \Longrightarrow(5)$ следует из леммы 1.10 , а импликация $(5) \Longrightarrow(1)$ очевидна.

Лемма 1.13. Пусть $A$ - риккартово справа конечномерное справа полупервичное кольцо. Тогда $A$ - полуравномерное справа кольцо.

ДоказАТЕЛЬСтво. Конечномерное справа кольцо $A$ обладает сушественным правым идеалом $B=\bigoplus_{i=1}^{n} B_{i}$, где все $B_{i}$ - ненулевые равномерные правые идеалы. По леммам 1.11 и 1.12 сушественньй правый идеал $B$ содержит регулярньй элемент $b$ кольца $A$. Так как $b A \cong A_{A}$, то достаточно доказать, что $b A_{A}$ - полуравномерный модуль. Для этого достаточно доказать, что если главный правый идеал $N$ кольца $A$ лежит в $B$ и имеет размерность Голди $m \leqslant n$, то $N$-полуравномерный модуль. При $m=1$ утверждение верно. Пусть $h_{i}: B \rightarrow B_{i}$ - естественные проекции. Так как все $h_{i}(N)$ - проективные модули, то $N \cap\left(\bigoplus_{j \neq i} B_{j}\right)$ - прямое слагаемое модуля $N_{A}$ для любого $i$, причем соответствуюшее дополнение изоморфно модулю $h_{i}(N)$. Теперь утверждение доказывается индукцией по числу $m$.

Лемма 1.14. Пусть $A$ - наследственное справа кольцо, $Q_{A}-$ инбективная оболочка модуля $A_{A}$. Тогда справедливо следующее:

(1) каждый инбективный правый $A$-модуль является вполне инвективныцм, а каждый проективный правый $A$-модуль является наследственнылм;

(2) любой ненулевой неразложимый инвективный модуль $E_{A}$ является гомоморфным образом модуля $Q$.

Пункт (1) леммы 1.14 доказан в $[1,39.16]$, а пункт (2) следует из (1) и леммы $1.9(1)$. 
Лемма $1.15[1,55.11]$. Для нётерова справа кольца А равносильны условия:

(1) $A$ - полуцепное кольцо;

(2) каждый конечнопорохсенный правый $A$-модуль является полуцепHbl.M;

(3) A - полуцепное справа кольцо, и каждый неразложимый инвективный правый $A$-модуль является иепным.

Лемма 1.16. Пусть $\left\{Y_{i}\right\}_{i \in I}$ - некоторое множество модулей. Тогда справедливо следующее:

(1) если все модули $Y_{i}$ проективны относительно некоторого модуля $M$, то $\bigoplus_{i \in I} Y_{i}-M$-проективный модуль;

(2) если $Y$ - подфактор модуля $\bigoplus_{i \in I} Y_{i}, X-$ модуль, проективный относительно всех модулей $Y_{i}$, причем либо $I$ - конечное множество, либо $X$ - конечнопорожденный модуль, то $X-Y$-проективный модуль;

(3) если $Y_{1}$ - квазипроективный модуль, то любая конечная прямая сумма изоморфных копий модуля $Y_{1}$ является квазипроективным модулем.

Пункты (1) и (2) леммы 1.16 доказаны в $[1,18.1,18.2]$, а (3) следует из (1) и (2).

Лемма 1.17. Пусть модуль $X$ проективен относительно модуля $Y$. Тогда справедливо следующее:

(1) если существует эпиморфизм $h: Y \rightarrow X$, то $\operatorname{Ker}(h)$ - прямое слагаемое модуля $Y$, причем $X$ - квазипроективный модуль, изоморфный прямому слагаемому модуля $Y$;

(2) если $X$ - t-порожденный модуль, где $t$ - кардинальное число, и существует такое натуральное число $n$, что модуль $Y^{n}$ содержит свободный подмодуль $F$ ранга $t$, то $X$ - проективный модуль;

(3) если $Y$ - конечно точный модуль, то модуль $X$ проективен относительно любого конечнопоро жденного правого $A$-модуля;

(4) если $X$ - конечнопорожденный модуль, а $Y$ - конечно точный модуль, то $X-$ проективный модуль;

(5) если $Y$ - неразложимий модуль, причем $X \neq 0$ и существует эпиморфизм $f: Y \rightarrow X$, то $f-$ изоморфизм.

ДоказАтельство. (1) Так как $X$ проективен относительно $Y$, то для эпиморфизма $f$ и тождественного отображения $1_{X}$ сушествует такой гомоморфизм $g: X \rightarrow Y$, что $1_{X}=f g$. Поэтому $Y=\bar{X} \oplus \operatorname{Ker}(f)$, где $\bar{X} \cong X$. Кроме того, $X$ проективен относительно $\bar{X}$. Поэтому $X$ - квазипроективный модуль.

(2) По лемме 1.16 (2) $X-F$-проективный модуль. Так как существует эпиморфизм $h: F \rightarrow X$, то по (1) модуль $X$ изоморфен прямому слагаемому свободного модуля $F$.

(3) Сушествует такое натуральное число $n$, что $Y^{n}$ содержит свободный циклический подмодуль $F$. По (1) $X-Y$-проективный модуль. По лемме 1.16 (2) $X$ - F-проективньй модуль. По лемме 1.16 (2) модуль $X$ проективен относительно любого конечнопорожденного свободного модуля. Так как произвольньй конечнопорожденный модуль $S$ является гомоморфньм образом конечнопорожденного свободного модуля, то по лемме 1.16 (2) $X$ проективен относительно любого конечнопорожденного модуля.

(4) Следует из (2).

(5) Следует из (1). 
Лемма $1.18[1,41.14]$. Пусть $M-\pi$-проективный модуль и $M=X \oplus Y$. Тогда справедливо следующее:

(1) $X, Y-\pi$-проективные модули, $X-Y$-проективный модуль, $Y$ $X$-проективный модуль;

(2) если $X \cong Y$, то $M$ - квазипроективный модуль.

Лемма 1.19. Пусть $A$ - несингулярное справа конечномерное справа первичное кольцо. Тогда А обладает простымм артиновым классическим правымм кольцом частных $Q$. Кроме того, справедливо следующее:

(1) $Q_{A}$ - инбективная оболочка модуля $A_{A}$ и $Q_{A}=Q_{1} \oplus \cdots \oplus Q_{n}$, где все $Q_{i}$ - изоморфные ненулевие несингулярные равномерные инбективные модули, причем каждый ненулевой несингулярный неразложимый инбективный $E_{A}$ изоморфен модулю $Q_{1}$;

(2) если существует ненулевой несингулярный конечномерный инбективный квазипроективный модуль $M_{A}$, то каждый ненулевой несингулярный конечномерный инбективный правый $A$-модуль является квазипроективным и $Q_{A}$ - квазипроективный модуль;

(3) если существует ненулевой несингулярный конечномерный инбективный неравномерный $\pi$-проективный модуль $M_{A}$, то каждый ненулевой несингулярный конечномерный инвективный правый $A$-модуль является квазипроективным и $Q_{A}-$ квазипроективный модуль;

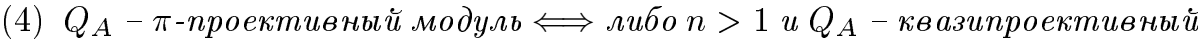
модуль, либо $n=1, A$ - равномерная справа область, $Q$ - правое тело частных области $A$, причем $Q_{A}-\pi$-проективньй модуль.

ДокАЗАТЕЛЬСТво. Пункт (1) следует из леммы 1.3 (3).

(2) Конечномерный модуль $M$ имеет ненулевое квазипроективное прямое слагаемое $E$. Теперь (2) следует из (1) и того, что по лемме 1.16 (3) каждая конечная прямая сумма изоморфных копий квазипроективного модуля является квазипроективным модулем.

(3) Так как $M$ - конечномерный неравномерный инъективный модуль, то $M$ имеет прямое слагаемое $E_{1} \oplus E_{2}$, где $E_{i}$ - неразложимые модули, являющиеся по (1) изоморфными. Так как $E_{1} \oplus E_{2}-\pi$-проективный модуль и $E_{1} \cong E_{2}$, то по лемме 1.18 (2) $E_{1}$ - квазипроективный модуль.

(4) Достаточно доказать импликацию $\Longrightarrow$. По (1) $Q_{A}=Q_{1} \oplus \cdots \oplus Q_{n}$, где все $Q_{i}$ - изоморфные несингулярные равномерные модули. Если $n=1$, то $A-$ равномерное справа несингулярное справа кольцо и, следовательно, равномерная справа область, $Q$ - правое тело частных области $A$, причем $Q_{A}-\pi$-проективный модуль. Пусть $n>1$. По (1) и (3) $Q_{A}$ - квазипроективный модуль.

Лемма 1.20. Пусть $A$ - наследственное справа нётерово справа полупервичное кольцо, $Q$ - полупростое артиново классическое правое кольцо частных кольца А. Тогда справедливо следующее:

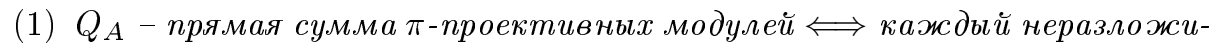

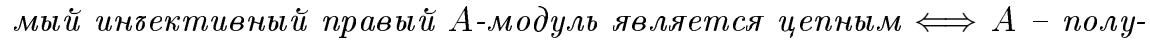
цепное кольцо;

(2) $J(A)=0$ и $Q_{A}-$ прямая сумма $\pi$-проективных модулей $\Longleftrightarrow A-$ простое артиново кольчо;

(3) $Q_{A}-\pi$-проективный модуль $\Longleftrightarrow$ либо $A-$ полуцепное кольцо и $Q_{A}-$ квазипроективный модуль, либо $A-$ чепная область. 
ДокАЗАТЕЛЬство. По лемме 1.12 А обладает полупростьм артиновым классическим правым кольцом частных $Q$, причем $Q_{A}$ - инъективная оболочка модуля $A_{A}$. По лемме 1.14 (1) $Q_{A}$ - вполне инъективный модуль. По лемме $1.13 \mathrm{~A}$ полуравномерное справа кольцо. Пункт (1) следует теперь из лемм $1.9(4), 1.15$ и того, что $Q_{A}$ - прямая сумма неразложимых инъективных модулей.

(2) Следует из того, что $A / J(A)$ - артиново кольцо, если $A$ - полуцепное кольцо.

(3) По (1) можно без ограничения общности считать, что $A$-полуцепное кольцо. По лемме 1.19 (4) остается рассмотреть случай, когда $A$ - полуцепная область. В этом случае утверждение следует из (1) и того, что полуцепная область является цепным кольцом.

Лемма 1.21. Пусть $Q$ - классическое кольцо частных наследственного нётерова первичного кольца А. Равносильны следующие условия:

(1) существует ненулевой несингулярный конечномерный индективный неравномерный $\pi$-проективный правый $A$-модуль;

(2) каждый ненулевой несингулярный конечномерный инбективный правый $A$-модуль является квазипроективнымм;

(3) $Q_{A}-$ квазипроективный модуль;

(4) существует такое натуральное число $n$, что кольцо $A$ изоморфно кольиу всех $(n \times n)$-матрии над некоторой полной чепной нётеровой областью $D$.

ДокАЗАТЕЛЬСтво. Эквивалентность условий (1), (2) и (3) следует из леммы 1.19 (1), (3).

$(3) \Longleftrightarrow(4)$. Эквивалентность условий (3) и (4) доказана в [2] в предположении, что $A$ не является примитивньм справа кольцом. Если выполнено условие (4) и $A$ является примитивным справа кольцом, то $A$ - простое артиново кольцо. Тогда вьполнено условие (3). Если выполнено условие (3) и $A$ является примитивньпм справа кольцом, то по лемме 1.20 (2) $A$ - простое артиново кольцо. Тогда выполнено условие (4).

ДоКАЗАТЕЛЬСТво ТЕОРЕМЫ 1. Пусть $Q$ - полупростое артиново классическое правое кольцо частных кольца $A$. По лемме $1.12 Q_{A}$ - инъективная оболочка модуля $A_{A}$. Импликация $(2) \Longrightarrow(1)$ следует из леммы $1.20(1)$. Докажем импликацию $(1) \Longrightarrow(2)$. Пусть $M$ - ненулевой инъективньй $\pi$-проективный правый $A$-модуль $E$, не являющийся сингулярным. Из леммы 1.1 (1) следует, что модуль $M$ содержит изоморфную копию ненулевого правого идеала $B$ кольца $A$. Без ограничения общности можно считать, что $B$ - ненулевой равномерный правый идеал, причем его инъективная оболочка $S$ является неразложимым $\pi$-проективным прямьм слагаемым модуля $Q_{A}$. По лемме 1.18 (1) $Q_{A}=Q_{1} \oplus \cdots \oplus Q_{n}$, где все модули $Q_{i}$ изоморфны неразложимому $\pi$-проективному модулю $S$.

ДокАЗАТЕЛЬСТво теОРемы 2. Теорема 2 следует из лемм 1.20 (3) и 1.21.

СлЕДСТвИЕ 1.22. Пусть $Q$ - классическое кольцо частных наследственного нётерова первичного кольца $A$.

Тогда $Q_{A}-\pi$-проективный модуль, не являющийся квазипроективным $\Longleftrightarrow A-$ чепная нётерова область, не являющаяся полной.

Следствие 1.22 вытекает из теоремы 2. 


\section{§2. Строение $\pi$-инъективных и $\pi$-проективных модулей}

ЛЕмма 2.1. Пусть модуль $X$ инвективен относительно модуля $Y,\left\{Z_{i}\right\}_{i \in I}$ - некоторое множество модулей. Тогда справедливо следующее:

(1) если все модули $Z_{i}$ ингективны относительно некоторого модуля $M$, то $\prod_{i \in I} Z_{i}-M$-инвективный модуль;

(2) если $Z$ - подфактор модуля $\bigoplus_{i \in I} Z_{i}, N$ - модуль, инвективный относительно всех модулей $Z_{i}$, то $N$ - Z-инвективный модуль;

(3) если $Z_{1}$ - квазиинбективный модуль, то любая конечная прямая сумма изоморфных копий модуля $Z_{1}$ является квазиинвективным модулем;

(4) если существует мономорфизм $f: X \rightarrow Y$, то $f(X)$ - прямое слагаемое модуля $Y$, причем $X$ - квазиинғективный модуль, изоморфный прямому слагаемому модуля $Y$;

(5) если $Y$ - конечно точный модуль, то $X$ - инвективный модуль;

(6) если существует мономорфизм $f: X \rightarrow Y$, то $f(X)$ - прямое слагаемое модуля $Y$, причем $X-$ квазиинвективный модуль;

(7) если $Y$ - неразложимый модуль, причем $X \neq 0$ и существует мономорфизм $f: X \rightarrow Y$, то $f-$ изоморфизм;

(8) если $M_{A}-$ модуль над кольцом $A$, причем для любого существенного правого идеала $B$ кольца $A$ кажсдий гомоморфизм $f: B_{A} \rightarrow M$ может быть продоләсен до гомоморфизма $g: A_{A} \rightarrow M$, то $M$ - инвективный модуль.

ДокАЗАТЕЛьство. Пункты (1) и (2) доказаны в $[1,16.1,16.2] ;(3)$ следует из (1) и (2). Доказательство (4) двойственно доказательству леммы 1.17 (1).

(5) Сушествует такое натуральное число $n$, что $Y^{n}$ содержит свободный циклический подмодуль $F$. По $(2) X-F$-инъективньй модуль. По критерию Бэра $[1,16.4] X$ - инъективный модуль.

(6) Следует из (4).

(7) Следует из (6).

(8) Пусть $\bar{B}$ - правый идеал кольца $A, \bar{f}: \bar{B}_{A} \rightarrow M$ - гомоморфизм. Найдется такой правый идеал $C$ кольца $A$, что $\bar{B} \cap C=0$ и правый идеал $B \equiv \bar{B} \oplus C$ является существенньм. Правило $f(\bar{b}+c)=\bar{f}(\bar{b})$ задает продолжение гомоморфизма $\bar{f}$ до гомоморфизма $f: B \rightarrow M$. По условию $f$ может быть продолжен до гомоморфизма $g: A_{A} \rightarrow M$. По (5) $M$ - инъективный модуль.

Лемма $2.2[1,41.20]$. Пусть $M-\pi$-инбективный модуль и $M=X \oplus Y$. Тогда справедливо следующее:

(1) $X, Y$ - т-ингективные модули, $X-Y$-инвективный модуль, $Y$ $X$-инбективный модуль;

(2) если $X \cong Y$, то $M-$ квазичнбективный модуль.

ЛЕМма 2.3. Пусть $T$ - сингулярный инвективный модуль, $U$ - несингулярный модуль, $M \equiv T \oplus U$. Тогда справедливо следующее:

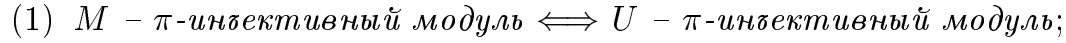

(2) если $U-$ - равномерный модуль, то $M-\pi$-ингективный модуль.

ДокАЗАТЕльство. Так как каждый равномерный модуль является $\pi$-инъективным, то достаточно доказать только пункт (1). Надо доказывать только импликацию $\Longleftarrow$. Пусть $\bar{U}$ - инъективная оболочка модуля $U, \bar{M} \equiv T \oplus \bar{U}$ - инъективная 
оболочка модуля $M, f$ - идемпотентный эндоморфизм модуля $\bar{M}$. По лемме 1.5 достаточно доказать вложение $f(M) \subseteq M$, что равносильно выполнению вложений $f(T) \subseteq T$ и $f(U) \subseteq T \oplus U$. Вложение $f(T) \subseteq T$ вытекает из того, что противном случае существовал бы ненулевой гомоморфизм из сингулярного модуля $T$ в несингулярный модуль $\bar{U}$, что противоречит лемме $1.2(1)$. Пусть $h: \bar{M} \rightarrow \bar{M} / T$ - естественный эпиморфизм. Так как $f(T) \subseteq T$, то $f$ индуцирует идемпотентньй эндоморфизм $g$ модуля $h(\bar{M})$. Так как $h(\bar{M}) \cong \bar{U}$ и $h(M) \cong U$, то $h(\bar{M})$ - инъективная оболочка $\pi$-инъективного модуля $h(M)$. По лемме $1.5 g(h(M)) \subseteq h(M)$. Поэтому $f(U) \subseteq T \oplus U$.

Лемма 2.4. Пусть $A$ - полупервичное правое кольцо Голди, $Q$ - полупростое артиново классическое правое кольцо частных кольиа $A, M$ - произвольнъй правый А-модуль. Тогда справедливо следующее:

(1) класс всех несингулярных правых $A$-модулей совпадает с классом всех правых А-модулей без кручения, а класс всех сингулярных правых A-модулей совпадает с классом всех периодических правых $A$-модулей;

(2) сингулярный подмодуль $\operatorname{Sing}(M)$ совпадает с периодической частью $T(M)$ модуля $M$

(3) фактормодуль $M / \operatorname{Sing}(M)$ является несингулярным модулем;

(4) если $M$ - существенное расширение сингулярного модуля, то $M$ сингулярный модуль;

(5) если $M$ - делимый несингулярный модуль, то $M$ - инбективный модуль;

(6) если $M$ - несингулярный редуцированный модуль, то для каждого ненулевого прямого слагаемого $N$ модуля $M$ существует такой регулярный әлемент $d \in A$, что $N \neq N d$.

ДокАЗАтЕльство. Пункт (1) следует из того, что по лемме 1.12 множество всех существенных правых идеалов кольца $A$ совпадает со множеством всех правых идеалов кольца $A$, содержащих регулярные элементы; $(2)$ следует из (1); (3) следует из (1) и (2); (4) следует из (3) и леммы 1.2 (2).

(5) По лемме 2.1 (8) достаточно доказать, что для любого существенного правого идеала $B$ кольца $A$ каждьй гомоморфизм $f: B_{A} \rightarrow M$ может быть продолжен до гомоморфизма $g: A_{A} \rightarrow M$. По лемме 1.12 сушественньй правый идеал $B$ содержит регулярный элемент $d$ кольца $A$. Так как $M$ - делимый модуль, то существует такой элемент $m \in M$, что $f(d)=m d$. Правило $g(x)=m x$ задает гомоморфизм $g: A_{A} \rightarrow M$. Пусть $b \in B$. Достаточно доказать, что $f(b)=m b$. Так как кольцо $A$ обладает классическим правым кольцом частных, то для элементов $b, d$ существуют такие элементы $a, t \in A$, где $t$ - регулярный элемент, что $b t=d a$. Тогда $f(b) t=f(d) a=m d a=m b t,(f(b)-m b) t=0$. Так как по (1) $M-$ модуль без кручения, то $f(b)=m b$. Поэтому $M$ - инъективный модуль.

(6) Из (5) и того, что каждый инъективньй подмодуль выделяется прямым слагаемым следует, что $M$ не имеет ненулевых делимых прямых слагаемых.

Лемма 2.5. Пусть $A$ - первичное правое кольцо Голди, $U_{A}-$ модуль, не являющийся сингулярным. Тогда справедливо следующее:

(1) $U$ - конечно точный модуль;

(2) любой $U$-инвективный модуль является инбективным модулем;

(3) любой U-проективный модуль является проективным относительно любого конечнопорожденного правого А-модуля; 
(4) любой U-проективный конечнопорожденный модуль является проективным модулем.

ДокАЗАТЕЛЬСТво. (1) По лемме 1.1 (1) модуль $U$ содержит изоморфную копию некоторого ненулевого правого идеала $N$ кольца $A$. Достаточно доказать, что $N_{A}$ - конечно точный модуль. Без ограничения общности можно считать, что $N$ - равномерный правый идеал. Конечномерное справа кольцо $A$ содержит существенный правый идеал $B=B_{1} \oplus \cdots \oplus B_{n}$, где все $B_{i}$ - ненулевые равномерные правые идеалы. По лемме 1.12 существенный правый идеал $B$ содержит регулярный элемент $b$. Поэтому $B$ содержит свободньй циклический $A$-модуль $b A$. По лемме $1.3(4)$ сушествуют мономорфизмы $B_{i} \rightarrow N$. Поэтому модуль $N^{n}$ содержит свободньй циклический подмодуль.

$(2),(3),(4)$ Следуют из (1), леммы 2.1(5) и леммы 1.17 (3), (4).

Лемма 2.6. Пусть $A$ - первичное правое кольио Голди, $M_{A}-\mathcal{м о д у л ь , ~ н е ~}$ являющийся сингулярным. Равносильны следующие условия:

(1) $M-\pi$-инвективный модуль;

(2) либо $M-$ инбективный модуль, либо $M=T \oplus U$, где $T$ - сингулярный инбективный модуль, $U$ - несингулярный равномерный модуль.

ДоКАЗАТЕЛЬСТво. Импликация $(2) \Longrightarrow(1)$ следует из леммы $2.3(2)$.

$(1) \Longrightarrow(2)$. Пусть $S \equiv \operatorname{Sing}(M), Q$ - инъективная оболочка модуля $M$. Тогда $Q=\bar{T} \oplus \bar{U}$, где $\bar{T}$ - инъективная оболочка модуля $S, \bar{U}$ - несингулярный модуль. По лемме 2.4 (4) $\bar{T}$ - сингулярный модуль. Пусть $T \equiv \bar{T} \cap M, U \equiv \bar{U} \cap M$. По лемме $1.5 M=T \oplus U$. Так как $T$ - сингулярный модуль и $M$ не является сингулярным модулем, то $U$ - ненулевой несингулярньй модуль. По лемме $2.2(1) T-$ $U$-инъективньй модуль. По лемме 2.5 (2) $T$ - инъективный модуль. Если $\bar{U}$ - неразложимый (инъективный) модуль, то $U$ - равномерный модуль. Допустим, что $\bar{U}=U_{1} \oplus \bar{U}_{2}$, где $\bar{U}_{i}$ - ненулевые модули. По лемме $1.5 M=U_{1} \oplus U_{2}$, где $U_{i} \equiv \bar{U}_{i}$. По лемме $2.2(1)$ ненулевые модули $U_{1}, U_{2}$ являются инъективньми относительно друг друга. По лемме $2.5(2) U_{1}$ и $U_{2}$ - инъективные модули. Поэтому $U_{i}=\bar{U}_{i}$, $M=Q-$ инъективньй модуль.

ЛЕМма 2.7. Пусть $Q$ - инбективная оболочка $\pi$-инбективного модуля $M$, причем $Q=\bigoplus_{i \in I} Q_{i}$. Тогда справедливо следующее:

(1) если $M \cap Q_{i}$ - вполне инвариантный подмодуль модуля $Q_{i}$ для любого

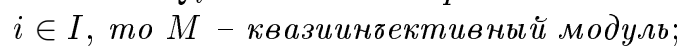

(2) если все $Q_{i}$ являются дистрибутивными полуартиновыми модулями,

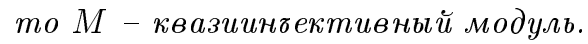

ДокаЗАТЕЛЬство. (1) Пусть $M_{i} \equiv M \cap Q_{i}, h_{i}: Q \rightarrow Q_{i}$ - естественные проекции. По лемме $1.5 M=\bigoplus_{i \in I} M_{i}$. В силу леммы 1.5 достаточно доказать, что $f(M) \subseteq M$ для любого $f \in \operatorname{End}(Q)$. Для этого достаточно доказать, что $\left(h_{j} f h_{i}\right)\left(M_{i}\right) \subseteq M_{j}$ для всех $i, j \in I$. По условию $\left(h_{i} f h_{i}\right)\left(M_{i}\right) \subseteq M_{i}$ для всех $i \in I$. Поэтому достаточно доказать, что $\left(h_{j} f h_{i}\right)\left(M_{i}\right) \subseteq M_{j}$ при $i \neq j$. Эндоморфизм $h_{j} f h_{i}$ модуля $Q$ индуцирует гомоморфизм $g: M_{i} \rightarrow Q_{j}$, причем $Q_{j}$ - сушественное расширение модуля $M_{j}$. Кроме того, по лемме $2.2(1) M_{j}-M_{i}$-инъективный модуль. По лемме $1.4(2)\left(h_{j} f h_{i}\right)\left(M_{i}\right)=g\left(M_{i}\right) \subseteq M_{j}$.

(2) Следует из (1) и того, что любой подмодуль дистрибутивного полуартинова модуля $Q_{i}$ является вполне инвариантным в $Q_{i}[6]$. 
Лемма 2.8. Пусть $Q_{A}-$ инбективный модуль над кольцом А. Тогда справедливо следующее:

(1) если $A$ - нётерово справа кольио, то $Q$ - прямая сумма неразложимых инбективных модулей;

(2) если $Q$ - несингулярный модуль и $A$ - конечномерное справа несингулярное справа кольио, то $Q$ - прямая сумма неразложсимых инвективных несингулярных модулей;

(3) если $Q$ - сингулярный модуль и $A$ - наследственное нётерово первичное кольцо, не являющееся примитивньм справа, то $Q-$ прямая сумма иепных артиновых инбективных счетнопорожсденных модулей, не являющихся конечнопорохсденными.

ДоказАтельство. Пункт (1) см. в $[1,27.4] ;$ (2) см. в [3, с. 84].

(3) По (1) и по лемме 2.4 (1) $Q$ - прямая сумма неразложимых периодических инъективных модулей. Теперь применяем полученное в [2, теорема 4] описание неразложимых периодических модулей над наследственными нётеровыми первичньми кольцами, не являющимися примитивными справа кольцами.

ЛЕмма 2.9. Пусть $M-\pi$-проективный модуль, причем каждый циклический подмодуль модуля $M$ является наследственным. Тогда справедливо следующее:

(1) если $M$ обладает максимальным подмодулем, то $M$ имеет ненулевое наследственное прямое слагаемое;

(2) если $M$ - конечномерный модуль, причем кажсдое ненулевое прямое слагаемое модуля $M$ обладает максимальным подмодулем, то $M$ проективный модуль.

ДокАЗАТЕЛьство. (1) Пусть $N$ - максимальньй подмодуль модуля $M, P-$ циклический подмодуль модуля $M$, не лежащий в $N$. Тогда $M=P+N$. Так как $M-\pi$-проективный модуль и $M=P+N$, то существует такой гомоморфизм $f: M \rightarrow P$, что $(1-f)(M) \subseteq N$. По условию $f(M)$ - проективный модуль. Поэтому проективньй модуль $f(M)$ изоморфен прямому слагаемому модуля $M$. Так как $M=f(M)+(1-f)(M)=f(M)+N \neq N$, то $f(M) \neq 0$.

(2) Следует из (1).

Лемма 2.10. Пусть $А$ - ограниченное слева полупервичное кольио Голди, $M_{A}$ - несингулярный модуль. Тогда справедливо следующее:

(1) если $M=M B$ для каждого ненулевого идеала $B$ кольца $A$, то $M$ инвективный модуль;

(2) если $M$ - редуцированный модуль, то для кажсдого ненулевого прямого слагаемого $N$ модуля $M$ существует такой ненулевой идеал $B$ кольиа $A$, что $N \neq N B$

(3) если $M$ - редуцированный модуль и кажсде собственное факторкольцо кольца $A$ является совершенным справа, то каждое ненулевое прямое слагаемое $N$ модуля $M$ обладает максимальным подмодулем.

ДоказАТЕЛьство. (1) По лемме 2.4(5) достаточно доказать, что $M=M d$ для любого регулярного элемента $d \in A$. Так как $A$ - полупервичное левое кольцо Голди, то по лемме $1.12 A d$-сушественный левьй идеал. По условию $A d$ содержит ненулевой идеал $B$ кольца $A$. По условию $M=M B$. Поэтому $M=M(A d)=M d$. 
(2) Следует из (1) и того, что каждьй инъективньй подмодуль является прямьм слагаемьм.

(3) По (2) существует такой ненулевой идеал $B$ кольца $A$, что $N \neq N B$. Так как каждый ненулевой правьй модуль над совершенным справа кольцом обладает максимальньм подмодулем $[7,22.8]$, то $(N / N B)_{A / B}$ обладает максимальным подмодулем. Поэтому $N_{A}$ обладает максимальным подмодулем.

ЛЕмма 2.11. Пусть $M_{A}$ - несингулярный конечнопорожсденный модуль над полупервичным кольцом Голди А. Тогда справедливо следующее:

(1) модуль $M$ изоморфен подмодулю свободного А-модуля;

(2) если $A$ - полунаследственное справа кольцо, то $M$ - проективный модуль;

(3) если $A$ - наследственное справа кольцо, то $M$ - наследственный модуль.

ДокАЗАТЕЛЬСтво. Пункт (1) см. в [3, с. 156]; (2) следует из (1) и того, что любой конечнопорожденный подмодуль проективного правого модуля над полунаследственным справа кольцом является проективным модулем [1, 39.13].

(3) Пусть $N$ - подмодуль модуля $M$. Так как $A$ - наследственное справа конечномерное справа кольцо, то $A$ - нётерово справа кольцо [3, с. 149]. Поэтому $N-$ конечнопорожденньй модуль. По (2) $N$ - проективньй модуль.

Лемма 2.12. Пусть А-наследственное нётерово первичное кольцо. Тогда справедливо следующее:

(1) каждое собственное факторкольцо кольца А является артиновым получепньм кольиом [7, 25.5.1];

(2) если $A$ не является примитивным справа кольцом, то $A$ - ограниченное кольцо [8].

Лемма 2.13. Пусть $А$ - ограниченное слева наследственное нётерово первичное кольцо, $M_{A}$ - несингулярный редуцированный модуль. Тогда справедливо следующее:

(1) каждое ненулевое прямое слагаемое $N$ модуля $M$ обладает максимальным подмодулем;

(2) если $M-\pi$-проективный модуль, то $M$ имеет ненулевое наследственное прямое слагаемое;

(3) если $M$ - конечномерный $\pi$-проективный модуль, то $M$ - проективнъцй модуль.

ДокАЗАТЕЛьство. Пункт (1) следует из 2.12 (1) и 2.10 (3). По 2.11 (3) каждый конечнопорожденньй подмодуль модуля $M$ является наследственным. Поэтому (2) и (3) следуют из (1) и из леммы 2.9.

Лемма $2.14[3,5.19]$. Пусть $M_{A}$ - проективный модуль над несингулярным справа кольцом $A$, причем $M$ - существенное расширение конечнопорожденного модуля.

Тогда $M$ - конечнопорожсенный модуль.

ДОКАЗАТЕЛЬСТВО ТЕОРЕМЫ 3. Если модуль $M_{A}$ не является сингулярным, то утверждение следует из леммы 2.6. Если $M$ - сингулярньй модуль, то утверждение следует из лемм 2.7 (2) и 2.8 (3). 


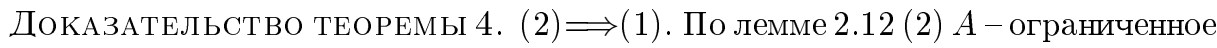
кольцо. По лемме 2.13(3) $M$ - проективный модуль. Так как любой конечномерный модуль является сушественным расширением конечнопорожденного модуля, то по лемме $2.14 M$ - конечнопорожденный модуль.

$(1) \Longrightarrow(2)$. Допустим, что конечнопорожденный модуль $M$ имеет ненулевое инъективное прямое слагаемое $E_{A}$. Можно без ограничения обшности считать, что $E$ - неразложимый инъективный конечнопорожденньй модуль. Пусть $Q$ - простое артиново классическое кольцо частных кольца $A$. По лемме 1.19 (1) модуль $Q_{A}$ является конечной прямой суммой изоморфных копий модуля $E$. Поэтому $Q_{A}$ - конечнопорожденный модуль. Пусть $Q=\sum_{i=1}^{n} q_{i} A$. Сушествуют такие элементы $t, a_{1}, \ldots, a_{n} \in A$, что $q_{i}=a_{i} t^{-1}$ при $i=1, \ldots, n$. Поэтому $Q=Q t=\sum_{i=1}^{n} a_{i} A \subseteq A \subseteq Q$. Тогда $A=Q$-простое кольцо. Получено противоречие с тем, что $A$ не является примитивным справа кольцом.

СлЕДСтвИЕ 2.15. Пусть $A$ - наследственное нётерово первичное кольцо, не являющееся ни примитивныц справа, ни полуцепным кольцом. Для модуля $M_{\text {А }}$ равносильны следуюшие условия:

(1) $M$ - конечнопорожденный проективный модуль;

(2) $M-\pi$-проективный конечномерный несингулярный модуль.

Следствие 2.15 вытекает из теорем 1 и 4 .

\section{Список литературы}

1. Wisbauer R. Foundations of module and ring theory. Philadelphia: Gordon and Breach, 1991.

2. Singh S. Quasi-injective and quasi-projective modules over hereditary noetherian prime rings // Canad. J. Math. 1974. V. 26. № 5. P. 1173-1185.

3. Goodearl K. R. Ring theory. Nonsingular rings and modules. New York: Marcel Dekker, 1976.

4. Stenström B. Rings of quotients: an introduction to methods of ring theory. Berlin: Springer-Verlag, 1975.

5. Фейс K. Алгебра: кольца, модули и категории. Т. 1. М.: Мир, 1977.

6. Stephenson $W$. Modules whose lattice of submodules is distributive // Proc. London Math. Soc. (3). 1974. V. 28. P. 291-310.

7. Фейс K. Алгебра: кольца, модули и категории. Т. 2. М.: Мир, 1979.

8. Lenagan T. H. Bounded hereditary noetherian prime rings // J. London Math. Soc. (2). 1973. V. 6. P. 241-246. 